\title{
A HEALTH-RELATED QUALITY OF LIFE AND PHARMACOTHERAPY COSTS STUDY FOR PATIENTS WITH CYSTIC FYBROSIS, GAUCHER DISEASE AND CHRONIC MYELOID LEUKEMIA IN BULGARIA
}

\author{
Maria Kamusheva ${ }^{1}$, Svetla Georgieva ${ }^{2}$, Dragana Lakic ${ }^{3},{\text { Konstantin } \text { Mitov }^{2}, \text { Alexandra Savova }^{2} \text {, Assena Stoimenova }}^{2}$ and Guenka \\ Petrova $^{2}$ \\ ${ }^{1}$ Medical University of Sofia, Faculty of Pharmacy, Sofia, Bulgaria \\ ${ }^{2}$ University Hospital "Alexandrovska”, Pharmacy Department, Sofia, Bulgaria \\ ${ }^{3}$ Belgrade University, Faculty of Pharmacy, Belgrade, Serbia \\ Correspondence to: Guenka Petrova \\ E-mail: guenka.petrova@gmail.com
}

\begin{abstract}
The aim of the present study was to analyze the cost of pharmacotherapy and quality of life of patients with cystic fibrosis (CF), Gaucher disease (GD) and chronic myeloid leukemia (CML) in Bulgaria. Data for the pharmacotherapy cost were compiled retrospectively. The second part of the study included a prospective quality-of-life survey (conducted at the Medical University Hospital "Alexandrovska" in 2010 and 2011). We estimated the average monthly pharmacotherapy costs according to data from the hospital registries. The quality-of-life survey was done through interviews based on the short form of SF-36, which measures 9 health domains. The total number of patients was 18, of which 10 were with CML, 5 with cystic fibrosis and 3 with Gaucher disease. The average age of the male and female patients with CML was 49.1 years and 48.3 years, respectively; 25 and 24 years in the group with GD, respectively; and 29.5 and 35.3 years in CF group, respectively. No statistical difference between the average monthly pharmacotherapy costs for the observed period was revealed. In all patients the quality of life was below the maximum possible value, which is equal to 100 points. The lowest values for the different indicators of quality of life were observed in the CF patients, most likely due to the chronic progression of this particular rare disease. The average range of quality of life for this group was 53.75. We found a statistical correlation between the pharmacotherapy costs and the quality of life. The Spearman correlation analysis revealed a correlation between the drug therapy costs and some quality-of-life indicators only in the patients with CML. The correlation between the pharmacotherapy costs and physiological functions in patients with $C M L$ was positive, i.e. higher costs improved this indicator. The pharmacotherapy costs were high, the average values for the three groups of patients being: BGN 9221.61 for 2010 and BGN 8254.64 for January-March 2011 for patients with CF, BGN 32954.08 for those with GD, and BGN 6389.38 for 2010 and BGN 6591.19 for the second period for the CML patients. The quality of life was significantly reduced for the observed patients: the average value for quality of life were 53.75, 65.03 and 67, respectively, from a maximum of 100.
\end{abstract}

Biotechnol. \& Biotechnol. Eq. 2013, 27(3), 3896-3900

Keywords: rare diseases, quality of life, cystic fibrosis, Gaucher disease, chronic myeloid leukemia

\section{Introduction}

Healthcare systems have two main health-related goals: increasing the quality of life and years of healthy life (13). Measuring the health-related quality of life (HRQoL) gives valuable information regarding the healthcare progress. HRQoL is a multi-dimensional concept that includes domains associated with physical, mental, emotional and social functioning. It goes beyond direct measures of population health, life expectancy and causes of death, and focuses on the impact that the health status has on the quality of life, evaluating the patient status before, during and after treatment (9). According to a study on the HRQoL in patients with rare diseases (chronic myeloid leukemia, Gaucher disease and cystic fibrosis), although some rare diseases do not affect life expectancy, most of them lead to physical, emotional and/or psychosocial disorders (14).

Rare diseases are pathological conditions that affect a relatively small group of the human population. These diseases are defined as life-threatening or chronically debilitating, severely reducing the quality and length of life $(1,10)$. Currently, there are between 6000 and 8000 distinct rare diseases (5860 of them are listed in the Orphanet database), affecting between $6 \%$ and $8 \%$ of the world population (1, $3)$. The governments in many countries, such as the USA, Australia, Japan and the European Union member-states, introduced specific legislation regulating the rare disease drugs and orphan drugs, indicated for treatment of rare diseases (1). Since its introduction in 1980, SF-36 became one of the most frequently used multifaceted measures of quality of life (4). Its application in patients with rare diseases is a suitable approach to estimate the quality of life, a fact confirmed by our study as well.

BIOTECHNOL. \& BIOTECHNOL. EQ. 27/2013/3 
Cystic fibrosis (CF) is an inherited rare disease of mucous and sweat glands. It affects mostly the lungs, pancreas, liver, intestines, sinuses, and reproductive organs and is a chronic progressive disease leading to multiorgan failure (7). The care for a child with CF is complex and Family Service Agencies are needed to help the family to solve different kind of problems connected with the disease (15).

Gaucher disease is an autosomal recessive lipid storage disorder cased by a deficient or defective production of lysosomal enzyme glucocerebrosidase (GD), resulting in an accumulation of glucocerebroside in the liver, spleen, bone marrow and rarely in the nervous system, lungs, kidneys, skin etc. Gaucher disease has three clinical subtypes and the most common one is the non-neuropathic form (type I), occurring in approximately 1 in 50000 live births $(11,18)$. The quality of life of patients with GD could be impaired because of debilitating clinical manifestations. A survey concludes that all eight health domains of SF-36 were improved after 2 years of treatment with Imiglucerase (17).

Chronic myelogenous leukemia (CML) is a blood cancer in which too many granulocytes are produced in the marrow (8).Clinical outcomes, are improved significantly after introduction of tyrosine kinase inhibitors (Imatinib, Dasatinib, Nilotinib), e.g. higher levels of complete cytogenetic response and improved survival (16).

The aim of our study was to analyze the costs of pharmacotherapy and quality of life of patients with cystic fibrosis, Gaucher disease and chronic myelogenous leukemia in Bulgaria. The study focused on the following questions: i. Is SF-36 applicable for estimation of the quality of life of patients with these three rare diseases in Bulgaria? ii. What are the main aspects of quality of life affected by the corresponding rare disease and what is their relationship to the cost of pharmacotherapy?

\section{Materials and Methods}

The study was conducted in University Hospital "Alexandrovska", which is the largest in Bulgaria, during 2010 and 2011. The data on pharmacotherapy costs were collected retrospectively. A prospective quality of life survey was conducted with the short version of the SF-36 form - a questionnaire introduced by McDowell, which is used to estimate the HQoL through nine multi-item dimensions covering physical activities, role limitations, bodily pain, social functioning, general mental health, role limitations due to emotional problems, vitality (energy or fatigue), general health perceptions and health status compared with the status reported for the previous year $(4,9)$. For each dimension, item scores were coded, summed, and transformed onto a scale from 0 (worst health) to 100 (best health), based on the patients' answers to 11 questions. The SF-36 was previously used and validated in Bulgaria for patients with chronic kidney diseases and after kidney transplantation (5). Five patients with CF, three patients with GD and ten patients with CML receiving BIOTECHNOL. \& BIOTECHNOL. EQ. 27/2013/3 their medicines from the hospital pharmacy at Hospital "Alexandrovska" were interviewed.

The average monthly pharmacotherapy cost for the three diagnoses were calculated based on data extracted from the hospital registries and covering the studied period: 2010 and January-March 2011.

Descriptive statistics, one-way ANOVA analysis and Spearman correlation analysis were performed to evaluate the statistical significance of the differences in the collected $\mathrm{HQoL}$ data.

\section{Results and Discussion}

\section{Pharmacotherapy costs for the monitored diseases}

Currently, there is no treatment for cystic fibrosis (CF). However, specialized pharmacotherapy with antibiotics, vitamins and other food supplements, enzymes, and inhalers, along with proper nutrition, can improve the quality and length of life for patients with CF (7). All patients with CF included in this study were treated with dornase alfa (highly purified solution of recombinant human deoxyribonuclease I [rhDNase]), inhaled tobramycin and enzymes (concentrated pancreatin-providing enzymes) for the entire period of the study. The patients with GD received enzyme replacement therapy with intravenous recombinant glucocerebrosidase (imiglucerase), and the treatment of patients with CML included BCR-ABL tyrosine kinase inhibitors: imatininb, dasatininb and nilotinib (for imatininb-resistant patients) (2, 12). Nilotinib, a new targeted drug developed to treat imatinibresitant patients with CML, was prescribed only to $10 \%$ of the affected patients in 2010 (Table 1).

The pharmacotherapy cost of patients with GD was associated with the highest value, followed by that of patients with $\mathrm{CF}$ and CML. No statistically significant difference was observed between the mean monthly cost of therapy per patient during the two observed periods: 2010 and January-March 2011 (Table 2).

\section{Quality of life}

All observed 18 patients were inquired with the short version of SF-36. The number of women and men did not differ significantly between the groups of patients. Ten patients were with CML, five with $\mathrm{CF}$ and three with GD. The percentage of male patients was $70 \%, 40 \%$ and $66.7 \%$ for each group, respectively. The female patients were $30 \%, 60 \%$ and $33.3 \%$, accordingly. The average age of the women with CF was 35 years and that of the men, 30 years. The average age of the patients with GD was 25 years, and 50 years for the CML patients.

In all patient groups, the average quality of life was lower than the maximum possible value of 100 points in all domains. The differences in the quality of life between the different diseases were statistically significant (ANOVA, $p=0.0076$ ). The patients with cystic fibrosis scored lower values for most indicators of quality of life: role limitations, bodily pain, social 
Relative share of prescribed medicines per diagnosis

\begin{tabular}{|l|l|l|l|l|}
\hline Condition & $\mathbf{2 0 1 0}$ & $\mathbf{2 0 1 1}$ & \multicolumn{2}{|l|}{} \\
\hline CF & dornase alpha, tobramycin, enzymes & $100 \%$ & dornase alpha, tobramycin, enzymes & $100 \%$ \\
\hline GD & imiglucerase & $100 \%$ & imiglucerase & $100 \%$ \\
\hline \multirow{3}{*}{ CML } & Imatinib & $60 \%$ & Imatinib & $50 \%$ \\
\cline { 2 - 5 } & Dasatinib & $30 \%$ & Dasatinib & $50 \%$ \\
\cline { 2 - 5 } & Nilotinib & $10 \%$ & & \\
\hline
\end{tabular}

TABLE 2

Mean monthly pharmacotherapy costs for the monitored diagnoses

\begin{tabular}{|l|l|l|l|}
\hline Condition & Mean monthly costs 2010 & $\begin{array}{l}\text { Mean monthly costs } \\
\text { Jan-Mar. 2011 }\end{array}$ & $\begin{array}{l}\text { One-way ANOVA } \\
(\boldsymbol{p})\end{array}$ \\
\hline CF & 9221.612 & 8254.639 & 0.516616 \\
\hline Gaucher disease & & 32954.08 & no \\
\hline CML & 6389.378 & 6591.19 & 0.750068 \\
\hline
\end{tabular}

TABLE 3

Descriptive statistics - cystic fibrosis and Gaucher disease

\begin{tabular}{|l|l|l|l|l|}
\hline & Minimum & Maximum & Mean & Std. deviation \\
\hline PhysFunc & 0.00 & 100.00 & 62.3309 & 37.66416 \\
\hline RoleLim & 0.00 & 100.00 & 44.4444 & 48.05234 \\
\hline Pain & 22.50 & 100.00 & 67.000 & 24.90607 \\
\hline SocAct & 22.50 & 100.00 & 70.8333 & 29.97395 \\
\hline PsychHealth & 32.00 & 92.00 & 71.5556 & 19.22961 \\
\hline EmotProb & 0.00 & 100.00 & 55.5444 & 47.14046 \\
\hline VitEnFat & 25.00 & 65.00 & 50.5556 & 13.33333 \\
\hline GenerHealt & 5.00 & 85.00 & 39.4444 & 26.27314 \\
\hline HealthPrYear & 50.00 & 100.00 & 66.6667 & 21.65064 \\
\hline
\end{tabular}

TABLE 4

Descriptive statistics - chronic myeloid leukemia

\begin{tabular}{|l|l|l|l|l|}
\hline & Minimum & Maximum & Mean & Std. deviation \\
\hline PhysFunc & 0.00 & 100.00 & 75.5100 & 30.65980 \\
\hline RoleLim & 0.00 & 100.00 & 77.5000 & 41.58325 \\
\hline Pain & 32.50 & 100.00 & 82.5000 & 24.77678 \\
\hline SocAct & 22.50 & 100.00 & 71.2500 & 27.03521 \\
\hline PsychHealth & 28.00 & 88.00 & 62.4000 & 21.09871 \\
\hline EmotProb & 0.00 & 100.00 & 76.6500 & 35.32041 \\
\hline VitEnFat & 30.00 & 65.00 & 46.0000 & 10.21981 \\
\hline GenerHealt & 30.00 & 90.00 & 55.0000 & 17.79513 \\
\hline HealthPrYear & 25.00 & 100.00 & 62,5000 & 21.24591 \\
\hline
\end{tabular}


Correlation between HQoL and pharmacotherapy costs

\begin{tabular}{|l|l|l|l|}
\hline Condition & Mean HQoL & Mean monthly costs 2011 & $\begin{array}{l}\text { One way ANOVA } \\
(\boldsymbol{p})\end{array}$ \\
\hline CF & 53.7484 & 8254.64 & \multirow{2}{*}{$p<0.05$} \\
\hline Gaucher disease & 65.0333 & 32954.08 & \\
\hline CML & 67.7011 & 6591.19 & \\
\hline
\end{tabular}

TABLE 6

Correlation between mean HQoL and pharmacotherapy costs

\begin{tabular}{|l|l|l|l|}
\hline Condition & Mean HQoL & $\begin{array}{l}\text { Mean monthly costs Jan- } \\
\text { Mar. 2011 }\end{array}$ & $\begin{array}{l}\text { Spearman correlation } \\
\text { coefficient }\end{array}$ \\
\hline CF & 53.7484 & 8254.639 & no \\
\hline Gaucher disease & 65.0333 & 32954.08 & no \\
\hline CML & 67.7011 & 6591.19 & $-0.706 ;$ \\
-0.697
\end{tabular}

TABLE 7

Correlation between the cost of therapy and QoL for the patients with CML

\begin{tabular}{|l|l|}
\hline Correlated variables & Spearman correlation coefficient \\
\hline Costs for pharmacotherapy and physiological functions & $0.673(0.05 \mathrm{CL})$ \\
\hline Costs for pharmacotherapy and pain management & $-0.706(0.05 \mathrm{CL})$ \\
\hline Costs for pharmacotherapy and mental health & -0.697 to $-0.803(0.05 \mathrm{CL})$ \\
\hline
\end{tabular}

functioning, role limitations due to emotional problems, and general mental health (Fig. 1, Table 3 and Table 4).

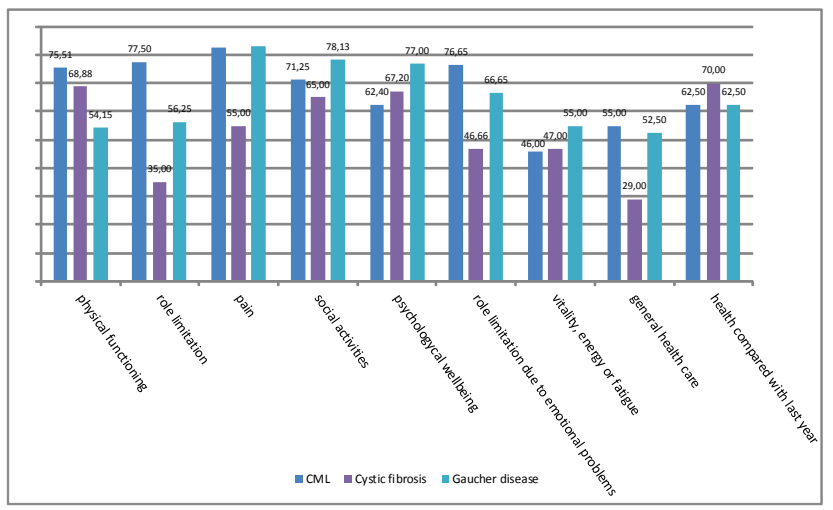

Fig. 1. Average value of SF-36 domains in the three studied groups of patients.

This can be explained by the fact that $\mathrm{CF}$ is a chronic progressive disease, significantly deteriorating the performance of daily activities for which there is only supportive care. The higher mean values of HQoL in patients with CML were probably due to the prescribed pharmacotherapy, which, as per the published scientific data, ensures long-term survival of over 5 years (6).

BIOTECHNOL. \& BIOTECHNOL. EQ. 27/2013/3
We found a correlation between the mean cost of pharmacotherapy and the mean HQoL in all three groups (Table 5), which showed that the quality of life depended on the amount of the pharmacotherapy costs. To determine what indicators of quality of life change when the costs of pharmacotherapy vary, we conducted a Spearman correlation analysis.

No correlations were found between the different aspects of quality of life and the patients' characteristics. Correlations existed only for particular quality characteristics, but they were logical, having in mind the interconnection of the survey questions, and they were not discussed either in the group of patients with CF, or in the group GD (Table 6).

In patients with CML, there was a positive Spearman correlation between the pharmacotherapy costs and physiological functions, i.e. the cost increase was found to improve this indicator, while a negative Spearman correlation was found between the pharmacotherapy costs and pain and mental health, which were obviously negatively affected (Table 6 and Table 7).

\section{Final remarks}

The results of our study give additional evidence for significantly reduced quality of life of patients suffering 
from rare diseases (CF, CML, GD) and high costs of pharmacotherapy associated with their treatment. The SF36 was found suitable for estimating the quality of life of patients with rare diseases: CML, GD and CF. With its implementation the health perception in a certain population could be measured and analyzed. All indicators of quality of life were scored as significantly affected in all three groups of patients. The patients with cystic fibrosis were those with the lowest values for most indicators, whereas the values of HRQoL were higher in the patients with CML. Based on our results, we also conclude that there is a correlation between physiological functioning and pharmacotherapy costs, between pain and pharmacotherapy costs and between mental health and pharmacotherapy costs in the patients with CML. Our study, however, has some limitations: e.g. the fact that it is not a long-term analysis but just a shot of the current state of the art. Further inquiries should be done to evaluate the long-term HQoL for patients with these three rare diseases in Bulgaria.

\section{Conclusions}

Our study indicates that, despite of the limited number of patients with rare diseases, the pharmacotherapy costs are high and there is a tendency for these costs to cause a significant burden to the health care budget. The study highlights the importance and specificity of three rare diseases, the need for targeted and sound finance of the expensive pharmacotherapy and for improvement of medical care for those patients whose quality of life is low and are in need of adequate and quality medical care.

To the best of our knowledge, this is the first Bulgarian study on the quality of life of patients with cystic fybrosis and Gaucher disease. It confirmed that the SF-36 could be successfully used in this group of patients as well as for comparison with the QoL of patients with chronic kidney disease.

\section{REFERENCES}

1. EUCERD (2010) 2009 Report on initiatives and incentives in the field of rare diseases of the European Union Committee of Experts on Rare Diseases (S. Aymé, C. Rodwell, Eds.) <http:// www.orpha.net/nestasso/EUCERD/upload/file/Reports/2009Rep ortInitiativesIncentives.pdf $>$
2. European Medicines Agency (n.d.) Cerezyme. Summary of product information < http://www.emea.europa.eu> (Accessed: Oct. 2012)

3. EURORDIS (2009) Orphan drugs: rising to the challenge to ensure a better future for 30 million patients in Europe, EURORDIS Statement, <http://www.eurordis.org/IMG/pdf/ Statement_Future_of_Orphan_Drugs_14_October_09.pdf $>$ (Accessed: Oct. 2012)

4. Frybach D., The Health Measurement Research Group, et al. (n.d.) Health-Related Quality of Life Measures <http://www. healthmeasurement.org/Measures.html> (Accessed: Sept. 2011)

5. Georgieva S., Kamusheva M., Lakić D., Mitov K., et al. (2012) Biotechnol. Biotech. Eq., 26(3), 3062-3065.

6. Hochhaus, A., Druker B., Sawyers C., Guilho F., et.al. (2008) Blood, 111, 1039-1043.

7. Kole A., Faurisson F., et al. (2009) The Voice of 12,000 Patients: Experiences and Expectations of Rare Disease Patients on Diagnosis and Care in Europe, Eurordis, pp. 123-129<http:// archive.eurordis.org>

8. Krustev Z. et al. (2003) In: Clinical Medicine for Pharmacists (Z. Krustev, Ed.), Ivan Sapundjiev-EOOD, Sofia, pp. 402-403. (In Bulgarian)

9. McDowell I. (2006) Measuring Health - A Guide to Rating Scales and Questionnaires, Oxford University Press, New York, pp. 649-650.

10. National Program (n.d.) Bulgarian National Program on Rare Diseases, 2009 - 2013 <http://www.mh.government.bg/ Articles.aspx ?lang=bg-BG\&pageid $=427 \&$ categoryid $=889>\quad$ (In Bulgarian)

11. Pastores, G., Weinreb, N., Aerts H., Andria G., et.al. (2004) Semin. Hematol., 41(Suppl. 5), 4-14.

12. Pena D.S. (2008) Oncology Business Review <www.oncbiz. com> (Accessed: Oct. 2012)

13. Petrova G., et al. (2010) Pharmacoeconomics, Infopharma, Sofia, p. 17. (In Bulgarian)

14. Rajmil L., Perestelo-Perez L., Herdman M. (2010) Adv. Exp. Med. Biol., 686, 251-272.

15. Turk J. (1964) Pediatrics, 34(1), 67-71.

16. Vorontsova A., Vinogradova O., et.al. (2010) Scientific Practical Journal Blood, 1(10), 22. (In Russian)

17. Weinreb N., Barranger J., Packman S., Prakash-Cheng A., et al. (2007) Clin. Genet., 71(6), 576-588.

18. Zimran A., Kay A., Gelbart T., Garver P., et al. (1992) Medicine, 71(6), 337-353. 